\title{
Opportunities and Challenges of Wireless Sensor Networks in Smart Grid
}

\author{
Vehbi C. Gungor, Member, IEEE, Bin Lu, Senior Member, IEEE, and Gerhard P. Hancke, Senior Member, IEEE
}

\begin{abstract}
The collaborative and low-cost nature of wireless sensor networks (WSNs) brings significant advantages over traditional communication technologies used in today's electric power systems. Recently, WSNs have been widely recognized as a promising technology that can enhance various aspects of today's electric power systems, including generation, delivery, and utilization, making them a vital component of the next-generation electric power system, the smart grid. However, harsh and complex electric-power-system environments pose great challenges in the reliability of WSN communications in smart-grid applications. This paper starts with an overview of the application of WSNs for electric power systems along with their opportunities and challenges and opens up future work in many unexploited research areas in diverse smart-grid applications. Then, it presents a comprehensive experimental study on the statistical characterization of the wireless channel in different electric-power-system environments, including a 500-kV substation, an industrial power control room, and an underground network transformer vault. Field tests have been performed on IEEE 802.15.4-compliant wireless sensor nodes in real-world power delivery and distribution systems to measure background noise, channel characteristics, and attenuation in the 2.4-GHz frequency band. Overall, the empirical measurements and experimental results provide valuable insights about IEEE 802.15.4-compliant sensor network platforms and guide design decisions and tradeoffs for WSN-based smart-grid applications.
\end{abstract}

Index Terms-CC2420, diagnostics, IEEE 802.15.4, link-quality indicator (LQI), monitoring, received signal strength indicator (RSSI), smart grid, wireless sensor networks (WSNs).

\section{INTRODUCTION}

$\mathbf{T}$ HE global climate change and rapidly growing populations over the past decades have generated increasing demands for abundant, sustainable, and clean electric energy on a global basis. However, in most countries today, the increasing energy demand means an even heavier burden on the already overstressed, overaged, and fragile electricity infrastructure. In the U.S., for example, the average age of power-grid transmission lines is beyond 50-60 years [29]. Over the last 20 years, the electricity demand and consumption have increased continuously by $2.5 \%$ annually according to a U.S. Department of

Manuscript received January 8, 2009; revised May 16, 2009 and June 27, 2009; accepted August 20, 2009. Date of publication February 5, 2010; date of current version September 10, 2010. The work of V. C. Gungor was supported by the European Union FP7 Marie Curie International Reintegration Grant (IRG) under Grant PIRG05-GA-2009-249206.

V. C. Gungor is with the Department of Computer Engineering, Bahçeşehir University, Istanbul 34353, Turkey (e-mail: cagri.gungor@bahcesehir.edu.tr).

B. Lu is with the Innovation Center, Eaton Corporation, Milwaukee, WI 53216-1897 USA (e-mail: binlu@ieee.org).

G. P. Hancke is with the University of Pretoria, Pretoria 0002, South Africa (e-mail: g.hancke@ieee.org).

Color versions of one or more of the figures in this paper are available online at http://ieeexplore.ieee.org.

Digital Object Identifier 10.1109/TIE.2009.2039455
Energy report [8]. The increasing electricity demand, together with the complex and nonlinear nature of the electric power distribution network, have caused serious network congestion issues. The network congestion and safety-related factors have become the main causes of several major blackouts that happened in recent years. In addition to the overstressed situation, the existing power grid also suffers from the lack of pervasive and effective communications, monitoring, fault diagnostics, and automation, which further increase the possibility of region-wide system breakdown due to the cascading effect initiated by a single fault. Furthermore, the global increasing adaptation of renewable and alternative energy sources in the 21st century also introduced new issues, such as power-grid integration, system stability, and energy storage, which also need to be addressed as additional challenges.

To address these challenges, a new concept of nextgeneration electric power system, a smart grid, has emerged. The smart grid is a modern electric power-grid infrastructure for improved efficiency, reliability, and safety, with smooth integration of renewable and alternative energy sources, through automated control and modern communication technologies [2], [5]. In the smart grid, reliable and online information becomes the key factor for reliable delivery of power from the generation units to the end users. The impact of equipment failures, capacity limitations, and natural accidents and catastrophes, which cause power disturbances and outages, can be largely avoided by online power system condition monitoring, diagnostics, and protection. In this respect, the intelligent and low-cost monitoring and control enabled by online sensing technologies have become essential to maintain safety, reliability, efficiency, and uptime of the smart grid [11], [13], [19], [30].

Traditional electric-power-system monitoring and diagnostic systems are typically realized through wired communications. However, the wired monitoring systems require expensive communication cables to be installed and regularly maintained, and thus, they are not widely implemented today because of their high cost [7]. Hence, there is an urgent need for cost-effective wireless monitoring and diagnostic systems that improve system reliability and efficiency by optimizing the management of electric power systems [11], [13].

As one of the main objectives, this paper gives a first glimpse and opens up future work in many unexploited research areas of applying wireless sensor networks (WSNs) in smart grid by providing an overview of the opportunities and challenges. The collaborative operation of WSNs brings significant advantages over traditional communication technologies, including rapid deployment, low cost, flexibility, and aggregated intelligence via parallel processing. The recent advances of WSNs have 
made it feasible to realize low-cost embedded electric utility monitoring and diagnostic systems [1], [4], [9], [11], [19], [30]. In these systems, wireless multifunctional sensor nodes are installed on the critical equipment of the smart grid and monitor the parameters critical to each equipment's condition. Such information enables the smart-grid system to respond to the changing conditions in a more proactively and timely manner. In this regard, WSNs play a vital role in creating a highly reliable and self-healing smart electric power grid that rapidly responds to online events with appropriate actions. The existing and potential applications of WSNs on smart grid span a wide range, including wireless automatic meter reading (WAMR), remote system monitoring, equipment fault diagnostics, etc. However, the realization of these currently designed and envisioned applications directly depends on efficient and reliable communication capabilities of the deployed WSNs [11], [13]. However, harsh and complex electric-power-system environments pose great challenges in the reliability of WSN communications in smart-grid applications.

Research activities related to the reliability of WSNs, such as wireless channel modeling and link-quality characterization, in harsh environments are extremely important when designing and deploying a reliable WSN in the smart grid. These channel models provide power system designers the ability to predict the performance of the communication network for a specific propagation environment, channel modulation, and frequency band. Although there exist radio propagation measurements in urban areas, office buildings, and factories [22], [28], [33], linkquality characterization in power generation, distribution, and industrial facility environments are yet to be efficiently studied and addressed.

To address this need, this paper presents a comprehensive experimental study on the statistical characterization of the wireless channel in different electric-power-system environments. Field tests have been performed on IEEE 802.15.4compliant wireless sensor nodes (using CC2420 radio chips) in real-world power delivery and distribution systems, including a $500-\mathrm{kV}$ substation, an industrial power control room, and an underground network transformer vault, to measure background noise, channel characteristics, and attenuation in the $2.4-\mathrm{GHz}$ frequency band. Various communication links, including both line-of-sight (LOS) and non-LOS (NLOS) scenarios, are also considered.

The remainder of this paper is organized as follows. In Sections II and III, the opportunities and challenges of applying WSNs in smart grid are reviewed, respectively. In Section IV, an overview of the related work on wireless link-quality measurements in WSNs is presented. The channel measurements and the field experiments are presented in Section V. Finally, this paper is concluded in Section VI.

\section{OpPortunities TO APPLY WSNs IN SMART GRID}

Electric power systems contain three major subsystems, power generation, power delivery, and power utilization. Recently, WSNs have been widely recognized as a promising technology that can enhance all these three subsystems, making WSNs a vital component of the next-generation electric power system, the smart grid. In this section, an overview of a few potential opportunities of applying WSNs in smart grid is discussed. Note that, depending on the application requirements, other wireless technologies, such as Wi-Fi and WiMAX, and power line communications can also be utilized in some of the smart-grid applications, such as in long-range backhaul communications in advanced metering applications.

\section{A. WAMR}

Recently, WSNs have provided a low-cost solution that enables WAMR systems for electric utilities. Overall, WAMR systems offer several advantages to electric utilities, including reduced electric utility operational costs by eliminating the need for human readers and online pricing models based on the online energy consumption of the customers [20] and for asset protection through advanced remote monitoring [11], [23]. However, WAMR systems require reliable two-way communications between electric utilities and the customer's metering devices. WSN technology addresses this requirement efficiently by providing low-cost and low-power wireless communications. Most utility and billing companies have recognized that, with the invention of low-cost low-power radio sensors, wireless communication is one of the most cost-efficient way to collect utility meter data.

\section{B. Remote System Monitoring and Equipment Fault Diagnostics}

In electric power systems today, safety and reliability have become the most critical tasks among all other tasks. System breakdown caused by component faults, environmental factors, or misoperation could cause huge economical losses and public concerns. For example, the August 2003 blackout that happened in U.S. and Canada left over 50 million people out of electricity and caused between 7 and 10 billion U.S. dollars of economical losses to the national economy for U.S and Canada [17]. Moving down to the facilities, the average unscheduled system downtime caused by unexpected equipment failures in various industries range from 70000 to 200000 U.S. dollars per hour [10]. It is commonly known that many of these power-grid and facility breakdowns could be avoided or at least largely alleviated if the critical system components are better monitored and the protection devices are better coordinated. However, the fact is that most of today's electric power infrastructure has only the minimal level of monitoring and automation and the majority of industries' critical equipment, such as motors less than $200 \mathrm{hp}$, are not even monitored. One of the main reasons of such dilemma is that the existing remote sensing, monitoring, and fault diagnostic solutions are still too expensive to be applied in a large-scale and pervasive basis.

With its low cost as one of the main advantages, WSNs provide a feasible and cost-effective sensing and communication solution for remote system monitoring and diagnosis systems. Efficient monitoring systems constructed by largescale deployment of smart sensor nodes can provide complete information on the conditions of system components, including generation units, transformers, transmission lines, motors, etc., 
in a remote and online manner [11], [12], [18], [24], [27]. With the online system monitoring and system-level coordinating controls and protections, a single system contingency in the power grid or facility could be detected and isolated before it causes cascading effects and results to more catastrophic system breakdowns.

\section{Challenges to ApPly WSNs IN SMART GRID}

The major technical challenges of WSNs in smart-grid applications can be outlined as follows.

1) Harsh environmental conditions. In electric-powersystem environments, the topology and wireless connectivity of the network may vary due to link failures. Furthermore, sensors may also be subject to RF interference, highly caustic or corrosive environments, high humidity levels, vibrations, dirt and dust, or other conditions that challenge performance [7], [13]. These harsh environmental conditions and dynamic network topologies may cause a portion of sensor nodes to malfunction or render the information they gather obsolete [11].

2) Reliability and latency requirements. The wide variety of applications envisaged on WSNs for smart grid will have different quality-of-service $(\mathrm{QoS})$ requirements and specifications in terms of reliability, latency, network throughput, etc. In addition, since sensor data are typically time sensitive, e.g., accidents in the electric power systems, it is important to receive the data at the controller node in a timely manner [11].

3) Packet errors and variable link capacity. In WSNs, the bandwidth of each wireless link depends on the interference level perceived at the receiver, and high bit error rates $\left(B E R=10^{-2}-10^{-6}\right)$ are observed in communication. In addition, wireless links exhibit widely varying characteristics over time and space due to obstructions and noisy environment in electric power systems. Hence, the bandwidth and communication latency at each wireless link are location dependent and can vary continuously. This makes it very hard to meet QoS requirements [11].

4) Resource constraints. The design and implementation of WSNs are constrained by three types of resources: a) energy; b) memory; and c) processing. In general, sensor nodes have limited battery energy supply [11]. For this reason, communication protocols for WSNs are mainly tailored to provide high energy efficiency.

Overall, in WSN-based smart-grid applications, a good linkquality metric is essential to a reliable and energy-efficient system operation. However, in harsh electric-power-system environments, rapid variations in the wireless channel preclude an efficient mechanism for knowing instantaneous link quality at the time of transmission, thus making it difficult to estimate the instantaneous value of the wireless link quality. In this paper, the focus of the field tests is on how to characterize wireless link quality in different electric power system environments based on a few radio-hardware measurements.

\section{RELATED WORK ON WIRELESS LINK ASSESSMENTS IN WSNS}

There have been numerous experimental studies on WSNs [25], [26], [31]-[33]. These studies have been performed either outdoors in open fields of different terrains or indoors in office settings and factory environments. In addition, the problem of coexistence between IEEE 802.11b and IEEE 802.15.4 networks has received a significant interest from the research community [3]. Different sensor platforms have been adopted, and each of them has had their respective radio protocols, frequency bands, channel modulation, and power levels. Despite their differences, their observations have mostly agreed on the following three aspects.

First, these studies have revealed the existence of three distinct reception regions in a wireless link: connected, transitional, and disconnected [31], [33]. The transitional region is often quite significant in size and is generally characterized by high variance in packet reception rates (PRRs).

Second, wireless link quality varies over space and time, deviating to a large extent from the idealized unit disk graph models used in network simulation tools [26], [33]. Based on these empirical studies and measurements, it is also found that the coverage area of sensor radios is neither circular nor convex, and packet losses due to fading and obstacles are common at a wide range of distances and keep varying over time.

Third, link asymmetry and radio irregularity were observed in several studies [25], [31]-[33]. Link asymmetry occurs when a node can successfully send packets to another node but not vice versa, even if both nodes are set to the same transmit power. In [32], the authors observed a significant percentage of radio links suffering asymmetry when the communication distance is large or the transmit power is low, which was confirmed by other researchers [25], [31], [33].

Although all these studies provide a valuable and solid foundation in WSNs, none of them addresses a statistical characterization of the wireless channel and link quality in indoor, outdoor, and underground electric-power-system environments [5], [26]. Consequently, the advances in sensor radio hardware as well as spatiotemporal link-quality variations in electric power systems call for new empirical measurements and analysis on IEEE 802.15.4-compliant sensor platforms deployed in different electric-power-system environments.

\section{Link-Quality MEASUREMENTS AND EXPERIMENTAL SETUP}

In this paper, we first focused on how to characterize and measure link quality in WSNs. We conducted experiments with Tmote Sky sensor nodes to obtain insights to answer this question. Tmote Sky nodes use the CC2420 radio component and support IEEE 802.15.4 [15]. Specifically, Tmote Sky motes operate in the $2.4-\mathrm{GHz}$ industrial, scientific, and medical band with an effective data rate of $250 \mathrm{~kb} / \mathrm{s}$. The higher data rate allows shorter active periods, further reducing energy consumption. Tmote Sky motes also integrate programming, computation, communication, and sensing onto a single device [21]. The integrated design provides an easy-to-use sensor mote 


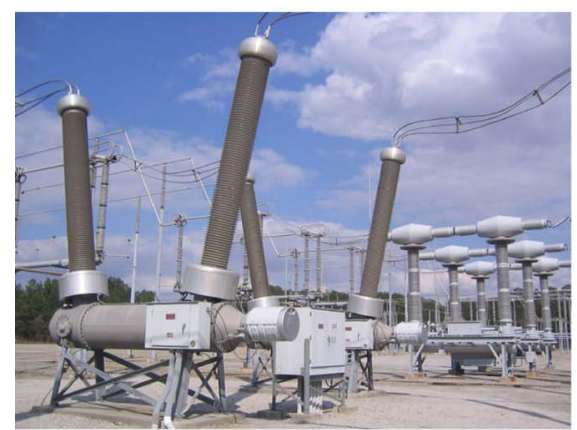

(a)

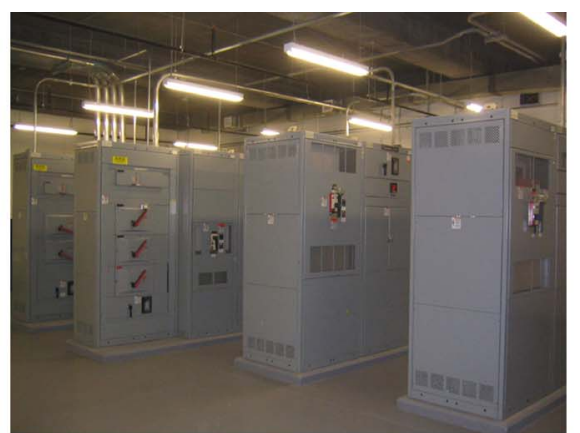

(b)

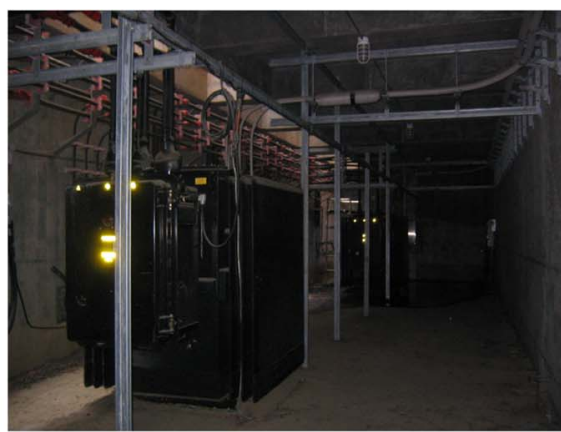

(c)

Fig. 1. Experimental sites. (a) Outdoor 500-kV substation environment, (b) indoor main power room, and (c) underground network transformer vault environments.

TABLE I

Mean Power Loss and Shadowing Deviation in Electric Power Environments

\begin{tabular}{|c|c|c|}
\hline Propagation Environment & $\begin{array}{c}\text { Path Loss } \\
(n)\end{array}$ & $\begin{array}{c}\text { Shadowing Deviation } \\
(\sigma)\end{array}$ \\
\hline 500 kv Substation (LOS) & 2.42 & 3.12 \\
\hline 500 kv Substation (NLOS) & 3.51 & 2.95 \\
\hline Underground Transformer Vault (LOS) & 1.45 & 2.45 \\
\hline Underground Transformer Vault (NLOS) & 3.15 & 3.19 \\
\hline Main Power Room (LOS) & 1.64 & 3.29 \\
\hline Main Power Room (NLOS) & 2.38 & 2.25 \\
\hline
\end{tabular}

with increased robustness, which is crucial for electric system automation applications. In our measurements, the packet length and buffer size are selected as 30 B and 64 packets, respectively.

Specifically, field tests have been performed to measure background noise, wireless channel characteristics, and attenuation in an indoor power control room, a 500-kV substation, and an underground network transformer vault. In Fig. 1, we present our experimental sites [20]. In these field tests, various communication links, including both line-of-sight (LOS) and non-LOS (NLOS) scenarios, are also considered.

\section{A. Wireless Channel Model}

In this paper, we modeled the wireless channel using a log-normal shadowing path-loss model through a combination of analytical and empirical methods. This model is used for large and small coverage systems, and moreover, experimental studies have shown that it provides more accurate multipath channel models than Nakagami and Rayleigh models for indoor wireless environments with obstructions [33]. In this model, the signal to noise ratio $\gamma(d)$ at a distance $d$ from the transmitter is given by

$$
\gamma(d)_{\mathrm{dB}}=P_{t}-P L\left(d_{0}\right)-10 \eta \log _{10}\left(\frac{d}{d_{0}}\right)-X_{\sigma}-P_{n}
$$

where $P_{t}$ is the transmit power in $\mathrm{dBm}, P L\left(d_{0}\right)$ is the path loss at a reference distance $d_{0}, \eta$ is the path-loss exponent, $X_{\sigma}$ is a zero mean Gaussian random variable with standard deviation $\sigma$, and $P_{n}$ is the noise power in $\mathrm{dBm}$.

In Table I, we present the radio propagation parameters for different electric-power-system environments. Note that, in Table I, the values of $n$ and $\sigma$ were calculated from the measured data in electric-power-system environments, using linear regression such that the difference between the measured and estimated path losses is minimized in a mean square error sense over a wide range of measurement locations and transmitter-receiver separations. These experiments were conducted over a period of several days for various locations and network configurations, including LOS and NLOS scenarios.

\section{B. Noise and Interference Measurements}

In this section, we first investigate the impact of background noise on the overall performance of 802.15.4 sensor networks in different electric-power-system environments. Then, we show the effect of electronic appliances on 802.15.4 sensor networks.

To measure background noise, we wrote a TinyOS application that samples RF energy at $62.5 \mathrm{~Hz}$ by reading the received signal strength indicator (RSSI) register of the CC2420 radio. The register contains the average RSSI over the past eight symbol periods. We sampled noise on different radio channels in a wide range of environments, including an indoor power control room, a 500-kV substation, and an underground network transformer vault. Fig. 2 shows our noise measurements and the effect of an electric appliance (microwave oven) on an 802.15.4 network. From the field measurements, the average noise level is found to be around $-90 \mathrm{dBm}$, which is significantly higher than that of outdoor environments, i.e., $-105-\mathrm{dBm}$ background noise is found in outdoor environments. We also observe that background noise continuously changes over time, which can be caused by temperature changes and interference levels. In Fig. 2(d), we also show the effect of microwave-oven interference on the noise floor measured by the Tmote Sky module [20]. As shown in Fig. 2, the interference from an existing microwave oven leads to a $15-\mathrm{dBm}$ interference in the $2.4-\mathrm{GHz}$ frequency band. 


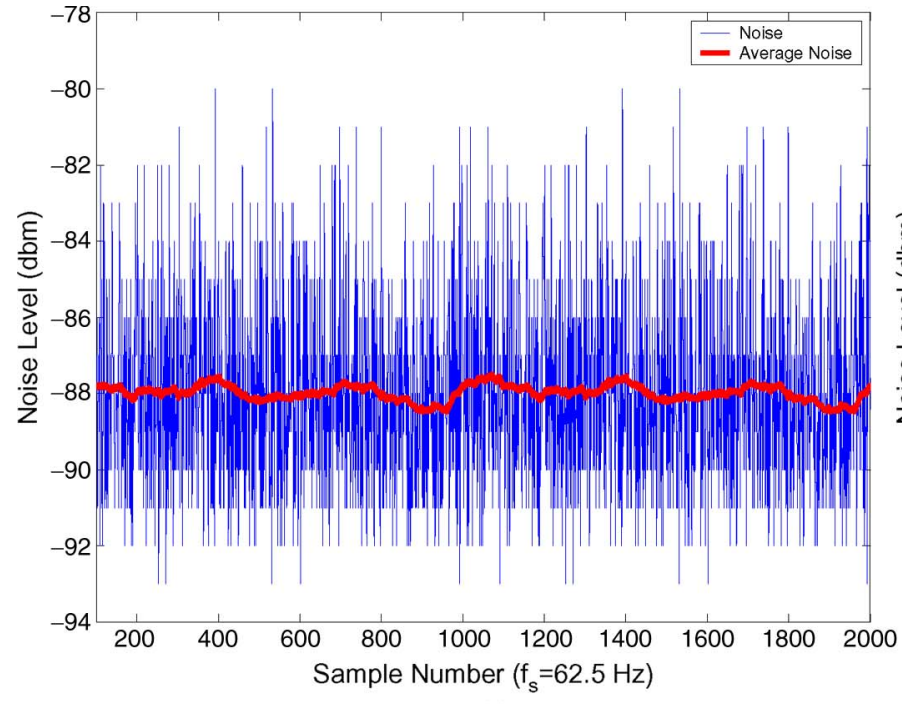

(a)

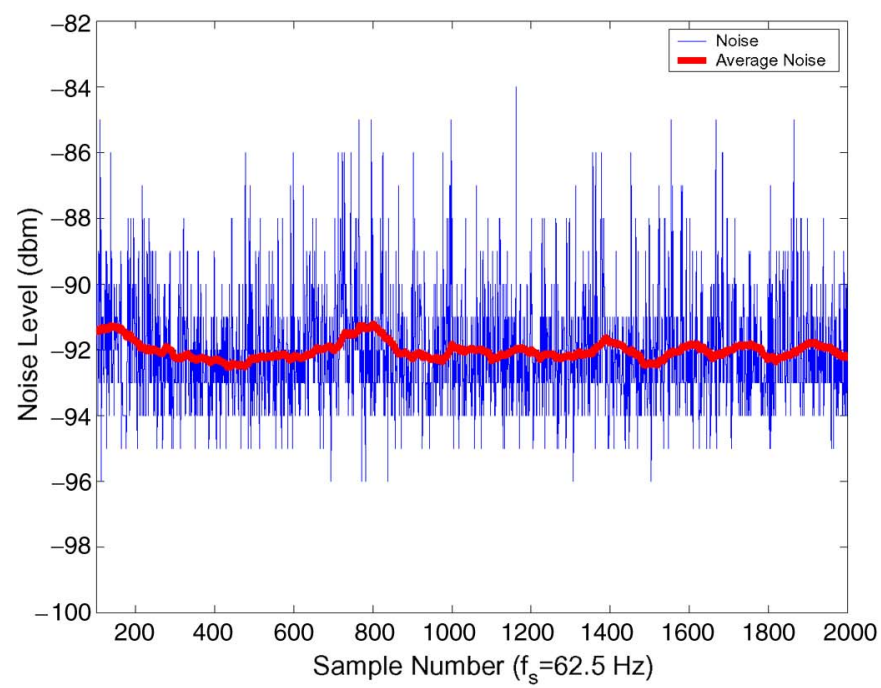

(c)

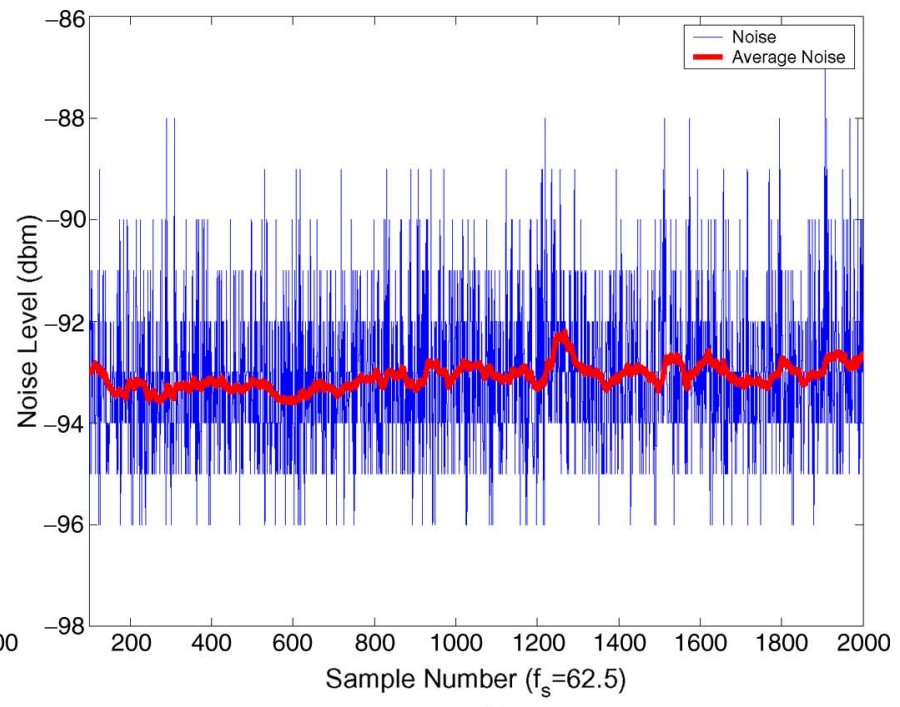

(b)

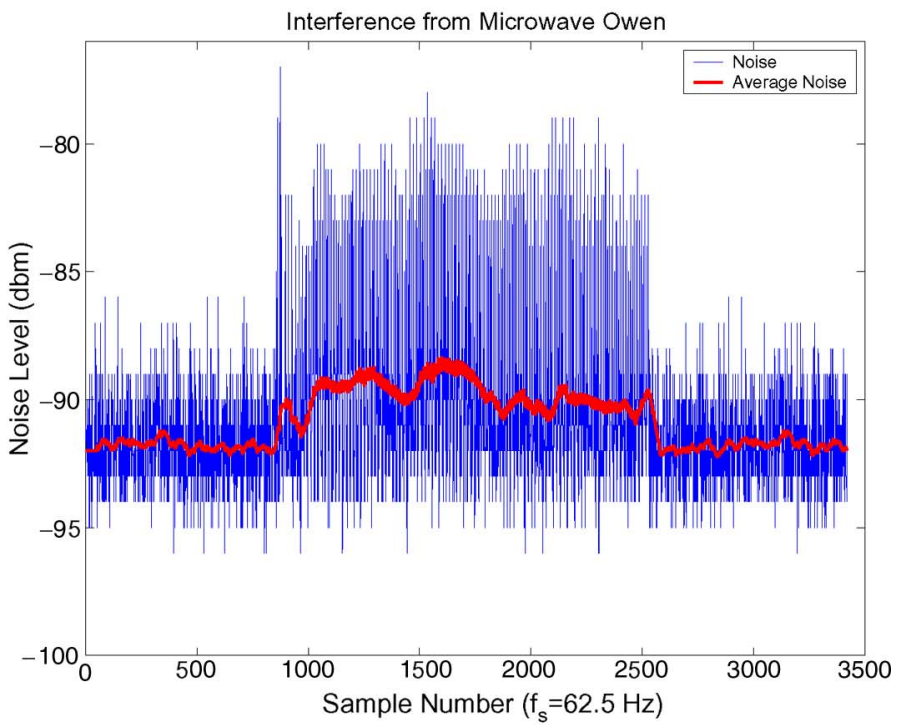

(d)

Fig. 2. Background noise measurements in (a) an indoor power control room, (b) a 500-kV substation, (c) an underground transformer vault, and (d) an indoor room, when a microwave oven is on.

To decrease the effect of noise and interference problems in the 2.4-GHz frequency band, Tmote Sky nodes use the direct sequence spread spectrum (DSSS) encoding scheme. To evaluate the DSSS's strength in real situations, we also measured the PRR when a pair of Tmote Sky motes are deployed close to a microwave oven operating in $2.4 \mathrm{GHz}$. The performance measurements show that the PRR varies from $35 \%$ to $100 \%$, when the microwave oven is turned on and off, respectively.

This experimental observation reveals that DSSS addresses the crowded spectrum issue in some degree, but is still far from enough. Here, it is important to note that some of the 802.15.4 frequencies overlap with $802.11 \mathrm{~b}$ frequencies, increasing the effects of external interference on link quality [3], [14]. In our performance measurements, we observe that only channel 26 in the 802.15 .4 spectrum is not affected by $802.11 \mathrm{~b}$ interference. Thus, to minimize $802.11 \mathrm{~b}$ interference in electric utility automation applications, e.g., WAMR systems, the default 802.15.4 channel can be set to 26. However, it is important to note that multichannel radios are often optimized for the center channel; therefore, selecting by default a channel at one extreme may significantly lower the radio performance. Hence, the utilities need to conduct large-scale field tests to decide the optimal channel for 802.15.4 sensor networks.

\section{Link-Quality Measurements}

In this section, we focused on how to characterize and measure link quality in sensor networks deployed in electric-powersystem environments. We have conducted experiments with Tmote Sky nodes. In our experiments, to measure the radio link quality, two useful radio-hardware link-quality metrics were used: LQI and RSSI. Specifically, RSSI is the estimate of the signal power and is calculated over eight symbol periods, while LQI can be viewed as chip error rate and is calculated over eight symbols following the start frame delimiter. LQI values are usually between 110 and 50 and correspond to maximum 

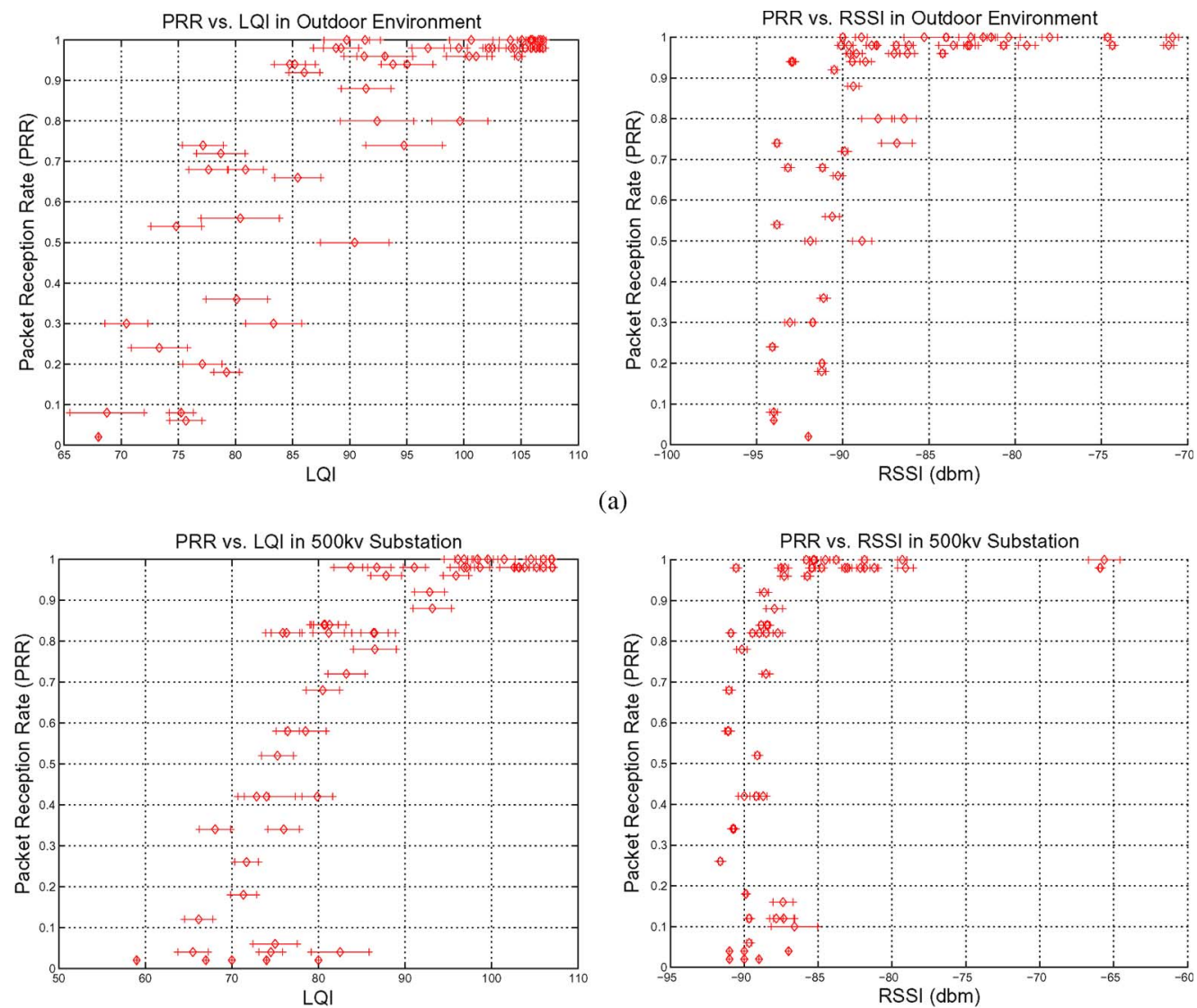

(a)

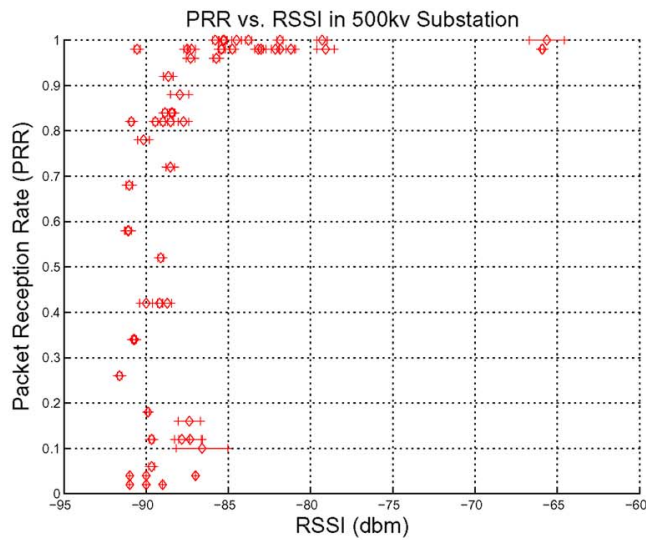

(b)
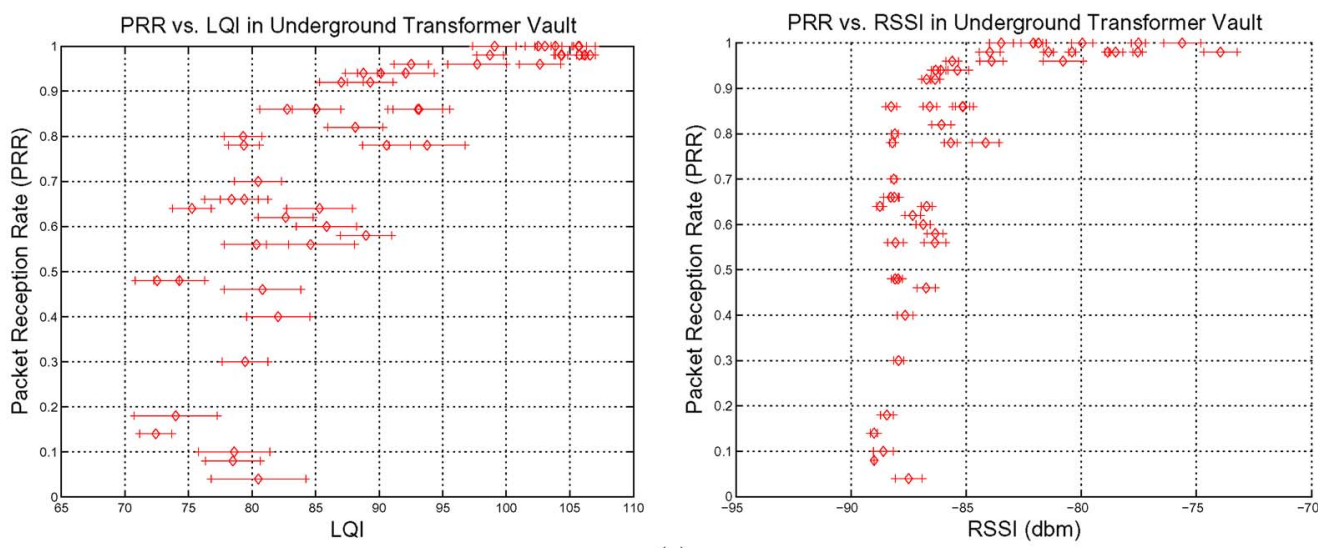

(c)
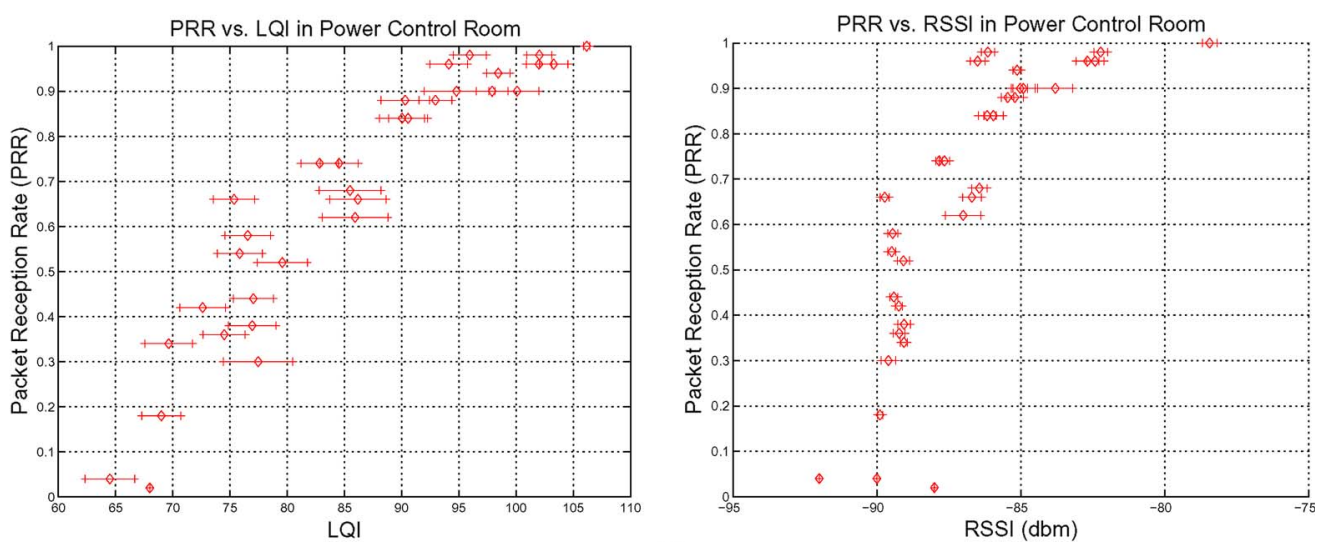

(d)

Fig. 3. PRR versus LQI and PRR versus RSSI measurements in (a) an outdoor environment, (b) a 500-kV substation, (c) an underground transformer vault, and (d) an indoor power control room. 
and minimum quality frames, respectively. The details of LQI metric can be found in the IEEE 802.15.4 standard [15].

In our experiments, we use a pair of Tmote Sky nodes in different utility environments and outdoor environment, one as the sender and the other as the receiver. We vary the distance from the receiver to the sender from 1 to $20 \mathrm{~m}$, in steps of $1 \mathrm{~m}$. The output power level of each sensor node and the packet size were set to be $-25 \mathrm{dBm}$ and $30 \mathrm{~B}$, respectively. At each distance, the transmitter sends 200 data packets with a rate of 2 packets/s. We deliberately chose a low rate to avoid any potential interference, so that the effect of unreliable links can be isolated from that of congestion. In addition, various NLOS communication links are also considered.

In Fig. 3, we present our preliminary experiment results to elaborate the relationship between PRR and link-quality metrics. Here, PRR represents the ratio of the number of successful packets to the total number of packets transmitted over a certain number of transmissions. Specifically, Fig. 3 shows 95\% confidence intervals. In this figure, we observe a strong correlation between the average LQI values and PRRs at the receiver. Statistical analysis shows that the Pearson correlation coefficient is around 0.70 between these two variables for the indoor main power room. This correlation implies that average LQI is a good measurable indicator of the packet reception probability. This observation is also consistent with the results in the related literature [20].

In Fig. 3, we also observe that there is a much smaller correlation between RSSI and the packet reception probability. The Pearson correlation coefficient is only 0.50 between the packet reception probability and the RSSI value. Furthermore, it is found that when the signal is weak [particularly when it is around the sensitivity threshold of CC2420(-94 dBm)], even though there is a considerable variation in the packet loss rate, RSSI does not provide any correlated behavior with PRR. Here, it is also important to note that all these measurements should be used as a reference. The exact values for a particular site are likely to vary depending on the actual environment propagation characteristics, RF interference, etc.

\section{CONCLUSION}

In this paper, a statistical characterization of the wireless channel in different electric-power-system environments has been presented. Field tests have been performed on IEEE 802.15.4-compliant sensor nodes (using CC2420 radio chips) in a $500-\mathrm{kV}$ substation, a main power control room, as well as an underground network transformer vault to measure background noise, channel characteristics, and attenuation in the $2.4-\mathrm{GHz}$ frequency band. Various communication links, including both LOS and NLOS scenarios, are also considered. In summary, the following major contributions have been made in this paper.

1) The potential applications of WSNs in smart grid are summarized. The WSN design and deployment challenges on wireless communication reliability are identified and experimentally studied.

2) The spatiotemporal impacts of electric-power-system environments on low-power wireless communications have been revealed. The relationship between the dynamics of link-quality and radio-hardware measurements has been identified based on extensive sets of link-quality measurements on IEEE 802.15.4-compliant sensor nodes (using CC2420 radio chips).

3) The empirical measurements in the field have demonstrated that average LQI values provided by WSN radio components are closely correlated with PRR and can be used as a reliable metric for wireless link-quality assessment during the operation of WSNs.

4) The wireless channel in different electric-power-system environments has been modeled using a log-normal shadowing path-loss model through a combination of analytical and empirical methods. Upon request, the complete experimental data will be made available. These can help the research community develop realistic link-quality models of WSNs for simulation-based studies.

Overall, the empirical measurements and experimental results in this paper provide valuable insights about IEEE 802.15.4-compliant sensor network platforms and guide design decisions and tradeoffs for WSN-based smart-grid applications. Future work includes developing adaptive and cross-layer communication protocols for smart grid applications.

\section{REFERENCES}

[1] I. F. Akyildiz, W. Su, Y. Sankarasubramaniam, and E. Cayirci, "Wireless sensor networks: A survey," Comput. Netw., vol. 38, no. 4, pp. 393-422, Mar. 2002.

[2] S. M. Amin and B. F. Wollenberg, "Toward a smart grid," IEEE Power Energy Mag., vol. 3, no. 5, pp. 34-41, Sep./Oct. 2005.

[3] L. Angrisani, M. Bertocco, D. Fortin, and A. Sona, "Experimental study of coexistence issues between IEEE 802.11b and IEEE 802.15.4 wireless networks," IEEE Trans. Instrum. Meas., vol. 57, no. 8, pp. 1514-1523, Aug. 2008.

[4] L. L. Bello, O. Mirabella, and A. Raucea, "Design and implementation of an educational testbed for experiencing with industrial communication networks," IEEE Trans. Ind. Electron., vol. 54, no. 6, pp. 3122-3133, Dec. 2007.

[5] U.S. Department of Energy, "The smart grid: An introduction," Washington, DC, Sep. 2008.

[6] U.S. Department of Energy, "Sensors and automation annual report," Office of Energy and Renewable Energy Rep., 2004.

[7] U.S. Department of Energy, "Assessment study on sensors and automation in the industries of the future," Office of Energy and Renewable Energy Rep., 2004.

[8] U.S. Department of Energy, "National transmission grid," Washington, DC, May. 2002.

[9] J. García, F. R. Palomo, A. Luque, C. Aracil, J. M. Quero, D. Carrión, F. Gámiz, P. Revilla, J. Pérez-Tinao, M. Moreno, P. Robles, and L. G. Franquelo, "Reconfigurable distributed network control system for industrial plant automation," IEEE Trans. Ind. Electron., vol. 51, no. 6, pp. 1168-1180, Dec. 2004.

[10] S. Grubic, J. M. Aller, B. Lu, and T. G. Habetler, "A survey on testing and monitoring methods for stator insulation systems of low-voltage induction machines focusing on turn insulation problems," IEEE Trans. Ind. Electron., vol. 55, no. 12, pp. 4127-4135, Dec. 2008.

[11] V. C. Gungor and F. C. Lambert, "A survey on communication networks for electric system automation," Comput. Netw., vol. 50, no. 7, pp. $877-$ 897, May 2006.

[12] V. C. Gungor, O. B. Akan, and I. F. Akyildiz, "A real-time and reliable transport protocol for wireless sensor and actor networks," IEEE/ACM Trans. Netw., vol. 16, no. 2, pp. 359-370, Apr. 2008.

[13] V. C. Gungor and G. P. Hancke, "Industrial wireless sensor networks: Challenges, design principles, and technical approaches," IEEE Trans. Ind. Electron., vol. 56, no. 10, pp. 4258-4265, Oct. 2009.

[14] I. Howitt and J. A. Gutierrez, "IEEE 802.15.4 low rate wireless personal area network coexistence issues," in Proc. IEEE Wireless Commun. Netw. 2003, pp. 1481-1486. 
[15] Wireless Medium Access Control (MAC) and Physical Layer (PHY) Specifications for Low-Rate Wireless Personal Area Networks (LR-WPANs), IEEE 802.15.4 Standard, Oct. 2003.

[16] H. Johal and D. Divan, "Design considerations for series-connected distributed FACTS converters," IEEE Trans. Ind. Appl., vol. 43, no. 6, pp. 1609-1618, Nov./Dec. 2007.

[17] S.-K. Joo, J.-C. Kim, and C.-C. Liu, "Empirical analysis of the impact of 2003 blackout on security values of U.S. utilities and electrical equipment manufacturing firms," IEEE Trans. Power Syst., vol. 22, no. 3, pp. 10121018, Aug. 2007.

[18] B. Lu, T. G. Habetler, R. G. Harley, J. A. Gutiérrez, and D. B. Durocher, "Energy evaluation goes wireless: Applying wireless sensor network in industrial plant energy evaluation and planning systems," IEEE Ind. Appl. Mag., vol. 13, no. 2, pp. 17-23, Mar./Apr. 2007.

[19] B. Lu and V. C. Gungor, "Online and remote motor energy monitoring and fault diagnostics using wireless sensor networks," IEEE Trans. Ind. Electron., vol. 56, no. 11, pp. 4651-4659, Nov. 2009.

[20] F. J. Molina, J. Barbancho, and J. Luque, "Automated meter reading and SCADA application for wireless sensor network," in Proc ADHOC-NOW, Montreal, QC, Canada, 2003, pp. 223-234.

[21] J. Polastre, R. Szewczyk, and D. Culler, "Telos: Enabling ultra-low power wireless research," in Proc. IEEE Inform. Process. Sensor Networks, Apr. 2005, pp. 364-369.

[22] T. Rappaport, Wireless Communications: Principles and Practice. Englewood Cliffs, NJ: Prentice-Hall, 2002.

[23] P. Rodriguez, A. V. Timbus, R. Teodorescu, M. Liserre, and F. Blaabjerg, "Flexible active power control of distributed power generation systems during grid faults," IEEE Trans. Ind. Electron., vol. 54, no. 5, pp. 25832592 , Oct. 2007

[24] M. Di Santo, A. Vaccaro, D. Villacci, and E. Zimeo, "A distributed architecture for online power systems security analysis," IEEE Trans. Ind. Electron., vol. 51, no. 6, pp. 1238-1248, Dec. 2004.

[25] D. Son, B. Krishnamachari, and J. Heidemann, "Experimental analysis of concurrent packet transmissions in low-power wireless networks," in Proc. ACM SENSYS, Nov. 2006, pp. 237-250.

[26] K. Srinivasan and P. Levis, "RSSI is under appreciated," in Proc EMNETs, Boston, MA, May 2006.

[27] H. Ukai, K. Nakamura, and N. Matsui, "DSP- and GPS-based synchronized measurement system of harmonics in wide-area distribution system," IEEE Trans. Ind. Electron., vol. 50, no. 6, pp. 1159-1164, Dec. 2003.

[28] A. Willig, M. Kubisch, C. Hoene, and A. Wolisz, "Measurements of a wireless link in an industrial environment using an IEEE 802.11compliant physical layer," IEEE Trans. Ind. Electron., vol. 49, no. 6, pp. 1265-1282, Dec. 2002.

[29] Y. Yang, D. Divan, R. G. Harley, and T. G. Habetler, "Power line sensornet-A new concept for power grid monitoring," in Proc. IEEE Power Eng. Soc. Gen. Meeting, Jun. 2006, pp. 1-8.

[30] Y. Yang, F. Lambert, and D. Divan, "A survey on technologies for implementing sensor networks for power delivery systems," in Proc. IEEE Power Eng. Soc. Gen. Meeting, Jun. 2007, pp. 1-8.

[31] J. Zhao and R. Govindan, "Understanding packet delivery performance in dense wireless sensor networks," in Proc. ACM SENSYS, Los Angeles, CA, 2003, pp. 1-13.

[32] G. Zhou, T. He, J. A. Stankovic, and T. Abdelzaher, "RID: Radio interference detection in wireless sensor networks," in Proc. IEEE INFOCOM, Miami, FL, Mar. 2005, pp. 891-901.

[33] M. Zuniga and B. Krishnamachari, "An analysis of unreliability and asymmetry in low-power wireless links," ACM Trans. Sensor Netw., vol. 3, no. 2, pp. 1-30, Jun. 2007.

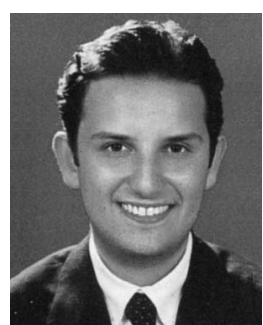

Vehbi C. Gungor (M'08) received the B.S. and M.S. degrees in electrical and electronics engineering from Middle East Technical University, Ankara, Turkey, in 2001 and 2003, respectively. He received the Ph.D. degree in electrical and computer engineering from the Broadband and Wireless Networking Laboratory, Georgia Institute of Technology, Atlanta, GA, USA, in 2007.

Currently, he is a faculty member in the Department of Computer Engineering, Bahcesehir University, Istanbul, Turkey. His current research interests are in wireless ad hoc and sensor networks, cognitive radio networks, IP networks, and smart grid applications. He has authored more than 30 papers in refereed journals and international conference proceedings and has been serving as a reviewer of numerous journals in these areas.

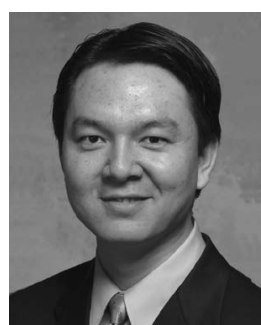

Bin Lu (S'00-M'06-SM'09) received the B.Eng. degree in automation from Tsinghua University, Beijing, China, in 2001, and the Ph.D. degree in electrical engineering from the Georgia Institute of Technology, Atlanta, in 2006.

Since October 2006, he has been with the Innovation Center of Eaton Corporation in Milwaukee, WI, where he is currently a Senior Engineering Specialist. His research interests include controls and diagnostics of electric machines and power electronics, computational intelligence applied to energy systems, and energy-efficient building technologies. He has published over 50 papers in refereed journals and conference proceedings and has 14 U.S. patent applications.

Dr. Lu was the recipient of the Second Prize Transactions Paper Award from the IEEE TRANSACTIONS ON INDUSTRY APPLICATIONS in 2008.

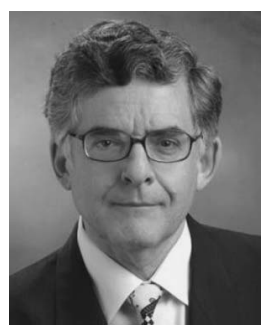

Gerhard P. Hancke (M'88-SM'00) received the B.Sc., B.Eng., and M.Eng. degrees from the University of Stellenbosch, Stellenbosch, South Africa, and the D.Eng. degree from the University of Pretoria, Pretoria, South Africa.

$\mathrm{He}$ has been engaged in engineering education, research, and management for more than 34 years. $\mathrm{He}$ is the Program Coordinator for Computer Engineering at the University of Pretoria and has played a major role in developing this program. He is the Head of the Advanced Sensor Networks Group and is collaborating in research projects internationally. He has published more than 100 papers in refereed journals and international conference proceedings. Before joining academia, he was a Senior Engineer with the Atomic Energy Board. 